\title{
REGULAR RIEMANNIAN $s$-MANIFOLDS OF NONCOMPACT TYPE
}

\author{
CRISTIAN U. SANCHEZ
}

\begin{abstract}
In this note it is proven that a regular Riemannian $s$-manifold of noncompact type (see below) cannot be immersed isometrically and equivariantly in $R^{n}$.
\end{abstract}

Our notation, terminology and basic facts will be those of [3].

Let $\left(M,\left\{S_{x}\right\}\right)$ be a connected periodic regular $s$-manifold which is metrizable, i.e. there is a Riemannian metric $g$ on $M$ which is invariant with respect to the symmetries $\left\{S_{x}: x \in M\right\}$. (Periodicity means that $\left(M,\left\{S_{x}\right\}\right)$ has finite order [3, p. 4].)

We have the group of isometries $I(M, g)$ which is transitive on $M[3$, p. 2].

Contained in $I(M, g)$ we have the group of transvections $G=\operatorname{Tr}\left(M,\left\{S_{x}\right\}\right)[3, \mathrm{p}$. 57] which is generated by the "elementary transvections", i.e. by the isometries $S_{x} \circ S_{y}^{-1}, x, y \in M$.

About the group $G$ one knows:

(1) $G$ is a connected Lie group [3, II 32, I 25].

(2) $G$ is transitive on $M[3$, II 33].

It is known [3, IV 24] that under the above conditions $\left(M,\left\{S_{x}\right\}\right)$ admits two complementary foliations $\widetilde{F}_{1}, \mathscr{F}_{2}$ such that:

(a) $\mathscr{F}_{1}$ is invariant and its leaves are regular $s$-manifolds with solvable group of transvections.

(b) $\mathcal{F}_{2}$ is weakly invariant and its leaves are regular $s$-manifolds with semisimple group of transvections (compare [2, p. 208]).

DEFINITION. We shall say that $\left(M,\left\{S_{x}\right\}\right)$ is of noncompact type if the foliation $\mathscr{F}_{2}$ has noncompact leaves.

The objective of this note is to prove the following.

TheOREM. Let $\left(M,\left\{S_{x}\right\}\right)$ be a connected periodic, regular s-manifold which is metrizable and of noncompact type. Then $\left(M,\left\{S_{x}\right\}\right)$ admits no isometric equivariant immersion into a finite-dimensional real representation of $G=\operatorname{Tr}\left(M,\left\{S_{x}\right\}\right)$.

Proof. Let us assume the existence of such an isometric immersion $(\varphi, f)$ : $(G, M) \rightarrow\left(I\left(R^{n}\right), R^{n}\right)$, where $\varphi$ is a Lie group monomorphism and $f$ is an isometric

Received by the editors January 8, 1982.

AMS (MOS) subject classifications (1970). Primary 53C40, 53B25.

Key words and phrases. Regular $s$-manifold, isometric equivariant immersion, Levi decomposition, 3-symmetric space. 
immersion such that

$$
f(g p)=\varphi(g) f(p) \quad \forall p \in M, g \in G .
$$

Let $p$ be a fixed point in $M$ and let $N(p)$ be an open neighborhood of $p$ in $M$ where $f$ is one-to-one.

Let us very briefly recall the definition of the foliation $\mathscr{F}_{2}$. Let $g$ be the Lie algebra of $G$.

Let $(V, S, \tilde{R}, \tilde{T})$ be the infinitesimal model of $\left(M,\left\{S_{x}\right\}\right)[3$, p. 74] and $(G, H, \sigma)$ the corresponding prime regular homogeneous $s$-manifold [3,p.53]. With the equivariant version of Levi's decomposition with respect to the compact group $K$ generated by $\operatorname{ad}(H)$ and $\sigma_{*}$ (the derivative of $\sigma$ at $e$ ), one may write $\mathfrak{g}=\mathfrak{r}+\mathfrak{s}$. Now one defines $W=\mathfrak{g} \cap V$; then it is "weakly invariant with respect to $\left(M,\left\{S_{x}\right\}\right)$ " [3, p. 92] and one defines a submanifold of $(V, S, \tilde{R}, \tilde{T}),\left(W, S^{\prime}, \tilde{R}^{\prime}, \tilde{T}^{\prime}\right), S^{\prime}=S \mid W$, $\tilde{R}^{\prime}=\tilde{R}\left|W, \tilde{T}^{\prime}=\tilde{T}\right| W$.

Then one uses [3, IV 7] with "weakly invariant with respect to $\left(M,\left\{S_{x}\right\}\right)$ " instead of invariant, which is true by [3, IV 10].

Let us go back to our proof. By using the above Levi decomposition we can obtain two analytic subgroups of $G, R$ for $\mathrm{r}$ and $Q$ for $\mathfrak{g} . R$ is the radical of $G$ and $Q$ is a maximal semisimple analytic subgroup of $G$ and $G=R \cdot Q[4$, p. 244].

It is easy to see from the definition of $\mathscr{F}_{2}$ and the action of $G[3, I 26]$ that the leaf of the foliation $\mathscr{F}_{2}$ which contains the point $p$ is precisely $Q(p)$ ( $p$ our chosen point). Then by our hypothesis $Q$ is noncompact.

Let $U(Q) \stackrel{\pi}{\rightarrow} Q$ be the universal covering of $Q$. Then $U(Q)=A_{1} \times \cdots \times A_{k} \times B_{1}$ $\times \cdots \times B_{l}$, where the $A_{i}$ 's are noncompact simple Lie groups, the $B_{j}$ 's are compact simple Lie groups, and $k \geqslant 1$.

Now for some of the $A_{i}$ 's, say $A_{1}$, the orbit $A_{1}(p)$ is nontrivial. Then it is a connected submanifold of dimension $\geqslant 1$.

Let us put $C_{p}=$ Connected component of $A_{1}(p) \cap N(p)$ containing $p$. Then we have:

(a) $f \mid C_{p}$ is one-to-one.

(b) $\varphi\left(\pi\left(A_{1}\right)\right)=\left\{\right.$ Id $\left.R^{n}\right\}[5]$.

(a) and (b) imply $C_{p}=\{p\}$. Then $\{p\}$ is open in $A_{1}(p)$ and therefore $A_{1}(p)=\{p\}$, which contradicts $\operatorname{dim} A_{1}(p) \geqslant 1$.

This result applies in particular to the 3-symmetric spaces classified by A. Gray in [1] and to the homogeneous regular $s$-manifolds $(G, H, \sigma)$ classified by A. Fedenko [3, p. 172].

For example we have

COROllaRY. If $M$ is a noncompact simply-connected indecomposable Riemannian 3-symmetric space and $G$ is the group of holomorphic isometries (it is transitive on $M$ ), then $M$ admits no isometric equivariant imbedding into a finite-dimensional representation of $G$.

For symmetric spaces this result is contained in [5].

ACKNOWLEdgement. We thank J. Vargas for bringing [5] to our attention. 


\section{REFERENCES}

1. A. Gray, Riemannian manifolds with geodesic symmetries of order 3, J. Differential Geom. 7 (1972), 343-369.

2. S. Helgason, Differential geometry and symmetric spaces, Academic Press, New York and London, 1962.

3. O. Kowalski, Generalized symmetric spaces, Lecture Notes in Math., vol. 805, Springer-Verlag, Berlin and New York, 1980.

4. V. S. Varadarajan, Lie groups, Lie algebras and their representations, Prentice-Hall, Englewood Cliffs, N. J., 1974.

5. J. Vargas, A symmetric space of noncompact type has no equivariant isometric immersion into Euclidean space, Proc. Amer. Math. Soc. 81 (1981), 149-150.

Instituto de Matemática, Astronomía y Física, Universidad Nacional de Cordoba, Avdas, Valparaiso y R. Martínez, 5000-CódDoba, ARgentina 